oil on the condenser and put the slide in contact with the oil. This slide may have a drop of syphilitic serum, or, for learning the method of dark-ground illumination, one may take a drop of stale urine or sewage, which is loaded with bacteria. Focus the object with the low power, then focus the condenser so that the image of the light source (say the edge of the flame) is on the same plane as the object, taking care that the cedar-oil maintains the connection between the slide and condenser. If the oil will not fill the gap, use a thicker slide, or use two thin slides, with a drop of oil between them, and another drop between the lower slide and the condenser. Now put the 4-millimeter objective on the microscope, focus the object with a small iris diaphragm, then open the diaphragm and put one of the center-stops under the condenser. Now change the focus of the condenser slightly if necessary, or shift the mirror a little, until the bacteria or other objects are bright on a dark background. Of course, it may be necessary to try several sizes of center-stops below the condenser, but once a certain size is found to work right, that one can be used regularly with that objective. I have used the above described method with a Leitz No. 7 objective, as well as a Spencer 4-millimeter, and while the results are not so good as with a reflecting condenser (on account of chromatic aberration), it has the merit of requiring no extra apparatus. My own preference, in searching for spirochetes, is to use an apochromatic dry objective and an achromatic oil-immersion condenser of English make, but an ordinary outfit will do the work fairly well.

To get the best results from any apparatus, one should know the principles underlying its use, and to get a good exposition of the general principles of dark-ground illumination, the books by Spitta and by Wright on microscopy are very valuable. But it is well to mention here that several amateur microscopists (that is, not medical men) of England, than whom there are no more skilful manipulators, have emphasized the danger of spurious images from dark-ground illumination. This does not apply to the spirochete, so much as to the other objects that may be viewed by this method.

1209 Walnut Street.

\section{ACUTE CHOLECYSTITIS COMPLICATING TYPHOID FEVER}

REPORT OF THREE CASES, WITH OPERATIONS

\section{E. M. PRINCE, M.D}

Gynecologist to St. Vincent's Hospital and Surgeon to Hillman Hospital

BIRMINGHAM, ALA.

The infrequency of reported cases in which cholecystitis has been observed as a complication of typhoid fever would lead one to believe this condition extremely rare, or that it might not have been looked for with the same vigilance as other complications. The post-typhoid cholecystitis, with at times calculus formation, has for some time been recognized as a frequent sequel of typhoid fever. Many of the cases must have been the result of an acute cholecystitis occurring during the attack of typhoid, the acute symptoms subsiding, leaving a chronic cholecystitis present. These patients with chronic cholecystitis are often operated on and the presence of the Bacillus typhosus demonstrated months or years after the attack of the fever. Then, if many sur- vive the acute cholecystitis, which very likely resulted from the typhoid attack, it is quite reasonable to think that there are many who die from this cause during the course of the fever.

I believe that acute cholecystitis, of sufficient severity to demand operative procedure, is of far more common occurrence than has heretofore been supposed, and the following cases may, therefore, be found of interest:

CASE 1.-History.-The patient, Mrs. S. B., aged 38, had had the usual diseases of childhood; otherwise she had always been healthy except for some stomach trouble during the previous six or eight years. She was taken ill on May 31, 1908, with pain in the abdomen, diarrhea and headache. She had complained of headache for the previous ten days, and had had some pain and tenderness in the abdomen, no appetite, and slight diarrhea.

Examination.-The tongue was red; the lungs normal; the abdomen slightly distended and tender. The spleen was enlarged; the temperature 100; the pulse 90. Blood examination: white blood count, 3,800; red blood count, 4,500,000; Widal negative; hemoglobin 95 (Tallquist). The urine was negative. Diagnosis, typhoid fever.

Course of Disease.-June 8: Spleen very much enlarged; white blood count 4,200; Widal positive. Temperature had continued to rise until at this time it was 103 to 104 in afternoon. Rose spots were very abundant. This case ran a course typical of typhoid fever for five weeks. The temperature and pulse became normal, remaining so for several days, when the patient again had a rise for several days. At this time the leucocyte count was 4,000 . The relapse lasted for several weeks and had about reached the normal on July 31 ; then the patient had a severe pain over the region of the gall bladder; the temperature rose to 105 , the pulse 170 to 180 . There was vomiting; the facial expression became very anxious; there was considerable tenderness over the region of the stomach and gall bladder. The blood showed at this time 11,000 leucocytes. (No differential count was made.) Diagnosis of a cholecystitis with perhaps perforation was made. I called in Dr. Lull, who con. curred in this, and while the chances from operation did not look very encouraging we earnestly insisted on this and gained the patient's consent after a few hours' wait. At this time (five hours later) the leucocyte count had risen to 15,000 , which was about 11,000 increase from a few days previous.

operation.-The patient was placed on the operating table about ten hours after the first pain was felt. I made an ineision over the gall bladder, and found this organ very much distended. On the fundus was a small necrotic area through which the very thick, dark-looking bile was being dischargeil. This bile was of the consistency of jelly, which accounts for the gall bladder not entirely emptying itself into the peritoneal cavity. Cholecystotomy was performed, using a rubber tube covered with a cigarette drain. I closed the gall bladder around this with a purse-string suture. The entire operation was completed and abdomen closed in eighteen minutes. The patient's condition seemed no worse than when the operation was commenced (which was bad enough). The patient was given hypodermoclysis on the table, and after the operation saline enemas, strychnin and spartein sulphate were given every three hours. In eighteen hours the temperature was 98.6, pulse 100 , and the patient made a rapid and uninterrupted recovery. The gall bladder discharged no bile until after the fourth day, when bile was freely discharged.

CASE 2.-History.-J. D. Almond, aged 23, was brought into the hospital on Sept. 1, 1908, the sixteenth day of typhoid fever. His previous history was good until the development of the present attack. When admitted to hospital the patient was suffering with severe pain over the gall bladder region, but had not complained of this until put on train to be brought into hospital.

Examination.-The eyes were normal; the tongue red and very dry; the heart and lungs normal; the spleen very much enlarged. There was tenderness over the gall bladder. The temperature was 103 ; the pulse 128 . The abdomen was distended and rigid. The patient was nauseated and had vomited 
a few times before admission. The Widal test was positive; white blood count 16,000 . Diagnosis was made of cholecystitis complicating typhoid fever.

operation.-The patient was carried to the operating room and an incision made over the gall bladder. This organ was found very much enlarged and distended. I opened the gall bladder, which was found to contain thick, dark bile. No stones were found. A rubber tube wrapped with cigarette drain was inserted, the edges of the gall bladder turned in and the tube retained by a purse-string suture. No bile was discharged for two days; then it began to discharge freely through the draining-tube. In twelve hours the temperature had dropped to 99.4 ; the pulse was 84 . The patient made a nice recovery. Culture from the bile was examined and a motile bacillus found.

CASE 3.-History.-Mrs. S. D., aged 15, was admitted to the hospital on Sept. 10, 1908, with diagnosis of typhoid fever. Her condition mentally was very bad and the history had to be obtained from her husband and the doctor bringing the case into the hospital. The past history could not be secured beyond the present illness. The patient had been ill one month; had been constantly in bed only for the previous five days, but had been having fever for two weeks past; the physician stated that the temperature ran up to 104 to 105 eacil afternoon and about 102 in the forenoon (when he had seen her). The pa. tient was four months pregnant. She was vomiting about once or twice each hour.

Examination.-The patient had a very anxious facial $3 x$ pression. The tongue was very red around the edges, with a dark coat, and trembled very markedly when protruded; it was hard to get the patient to protrude the tongue on account of her bad mental condition. The chest seemed normal. The spleen could not be felt on account of the distended abdomen and extreme tenderness. There was much tenderness over the gall bladder and some tenderness over the appendix and uterus. The patient seemed in great pain. The blood examination showed white blood count 4,000 , hemoglobin 40 per cent., Widal very positive. The pulse was 140 , the temperature 103.4. The urine showed albumin present; there were abundant hyaline and granular casts. The diagnosis was made of typhoid fever complicated by suppurative cholecystitis.

Operation was advised, but the husband was unwilling to have the patient operated on until her mother could be consuited. We offered no encouragement and did not insist on operating. The mother did not arrive until the day following, and gave her consent to operation. At this time the patient's condicion did not seem much changed for the worse, as it had been about as bad as possible on the day of admission. According to the nurse's notes, between $7 \mathrm{p}$. m., September 10 and 7 a. m., September 11, the patient was delirious, suffering great pain in the abdomen. She had five liquid movements during this period. Three-quarters of a grain of morphin sulphate was given by hypodermic injection during the night. It will be seen that we did not have a good subject for a surgical operation. The temperature at this time was 104.6, pulse 132, white blood count 10,000 .

Operation.-An incision was made over the gall bladder, ex posing the firmly adherent omentum covering the organ. The omentum was dissected away, exposing a very much distended gall bladder. The liver was speckled or nutmeg in appearance. The gall bladder, on being opened, was found to contain a thick, tarry bile mixed with a yellowish discharge, which proved to be pus. The gall bladder was drained after a culture was taken for bacteriologic examination. On account of pain in the lower abdominal region we considered it best to explore this region. An incision was made in median line; the uterus, being brought into view, presented a very unhealthy appearance. The question of a living or dead fetus was undecided, and considering that we had perhaps a toxemia of preg. nancy, a dead fetus and a very unhealthy uterus, we decided to remove it. The appendix was normal. The time consumed in removing the uterus, draining the gall bladder and closing both incisions was eighteen minutes. The patient left the table in about as good condition as when she came on.

Postoperative History.-On the third day there was a normal movement from tıe bowels; the temperature and pulse began to fall until on the eighth day after the operation the pulse had reached 100 and the temperature 100.8 by rectum. The bowels were acting nicely and the prospects for continued improvement seemed bright, when the patient's condition became suddenly worse. The temperature per rectum was 98 , the pulse 144, and there was great pain in the right iliac region. Drs. Johnson and Whelan were calied in and the diagnosis of perforation was made, but on account of the desperate condition of the patient operation was not advised. The diagnosis was found correct at autopsy, the perforation being about four inches from the ileocecal junction. The report on the gallbladder findings was a mixed infection of a motile bacillus and streptococcus:

1091/2 North Twentieth Sireet.

\section{Therapeutics}

\section{INFANT FEEDING}

In this age of bottle-fed infants, milk foods, substitute foods, pasteurized milk, and modified milk, it may be well to ponder some on the facts presented in a paper by Dr. Joseph E. Winters, of New York, Professor of Diseases of Children at the Cornell University Medical College. It may be well to present his findings in the form of aphorisms.

1. Infant mortality has only lately decreased, while the mortality of older individuals has been continuously decreasing.

2. A breast-fed child in the worst surroundings has an immunity from disease never found under any condition of substitute feeding.

3. The mother's milk is very rich in fat, containing 5 per cent. (together with 7 per cent. of milk sugar, and 1.25 per cent. of proteid) and, other things being equal, the fatter the child, the healthier it is.

4. The extra amount of fat which the child receives from its mother's milk over and above that from: artificially prepared foods is absolutely necessary for the maintenance of its requisite heat, and when it is remembered that heat is largely lost by radiation and evaporation from the surface of the body, and the child having relatively three times as much surface for radiation and evaporation as the adult, the importance of maintaining the fat content of its food is at once seen.

5 . The extra amount of fat is also needed for the child's nutrition on account of the rapid growth of brain, nerves, and bone marrow, all of which tissues contain large amounts of fat.

6. Separator (or centrifugal) cream is prepared from whole milk by rapid revolutions of the separator, and is consequently lower than gravity cream in all of its constituents except fat, and would be more correctly named if called centrifugal or separator butter fats instead of cream. Also, by the excessive pressure the fat globules are no longer free, but are so crushed together that when added to modified milk they form masses of fat that can not be well digested by the child. Such conglomerate fat which must reach the intestine for digestion, "does not readily pass through the narrow pylorus of the infant. Such fat is, then, as indigestible as cheese."

7. "Centrifugal cream is almost proteid-free and nearly destitute of growth constituents, hence the only food value it can have is as an addition of fat to other milk."

8. The upper half-ounce of gravity cream contains 3 per cent. of proteid, and gives fat globules so loosely 